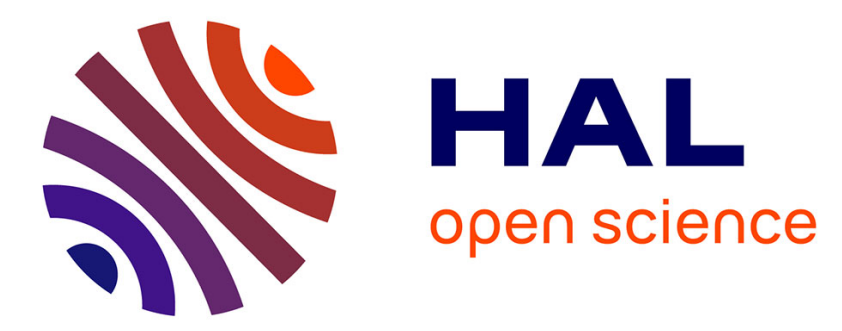

\title{
Error estimate for a 1D-2D finite volume scheme. Comparison with a standard scheme on a 2D non-admissible mesh.
}

Marie-Claude Viallon

\section{- To cite this version:}

Marie-Claude Viallon. Error estimate for a 1D-2D finite volume scheme. Comparison with a standard scheme on a 2D non-admissible mesh.. Comptes rendus hebdomadaires des séances de l'Académie des sciences, 2013, 351, pp.47-51. 10.1016/j.crma.2013.01.011 . hal-00916774

\section{HAL Id: hal-00916774 \\ https://hal.science/hal-00916774}

Submitted on 10 Dec 2013

HAL is a multi-disciplinary open access archive for the deposit and dissemination of scientific research documents, whether they are published or not. The documents may come from teaching and research institutions in France or abroad, or from public or private research centers.
L'archive ouverte pluridisciplinaire HAL, est destinée au dépôt et à la diffusion de documents scientifiques de niveau recherche, publiés ou non, émanant des établissements d'enseignement et de recherche français ou étrangers, des laboratoires publics ou privés. 


\title{
Error estimate for a 1D-2D finite volume scheme. Comparison with a standard scheme on a 2D non-admissible mesh.
}

\author{
Marie-Claude Viallon ${ }^{\mathrm{a}}$ \\ a Université de Lyon, UMR CNRS 5208, Université Jean Monnet, Institut Camille Jordan, Faculté des Sciences et \\ Techniques, 23 rue Docteur Paul Michelon, 42023 Saint-Etienne Cedex 2, France. \\ Received $* * * * * ;$ accepted after revision +++++ \\ Presented by
}

\begin{abstract}
We study an hybrid finite volume scheme to solve a problem set in a domain consisting of several zones of different dimensions in space. For a linear 1D-2D model problem, we define a specific $H^{1}$ discrete norm and we state an error estimate in this norm. We compare the hybrid scheme to a classical scheme used on a 2D non-admissible mesh. To cite this article: A. Name1, A. Name2, C. R. Acad. Sci. Paris, Ser. I 340 (2005).
\end{abstract}

Résumé

Estimation d'erreur pour un schéma volumes finis 1D-2D. Comparaison avec un schéma standard sur un maillage 2D non-admissible. On étudie un schéma volumes finis hybride pour résoudre un problème posé dans un domaine où la dimension en espace est différente d'une zone à l'autre. Pour un problème modèle linéaire 1D-2D, nous définissons une norme $H^{1}$ discrète 1D-2D adaptée, et nous établissons une estimation d'erreur dans cette norme. Nous comparons le schéma hybride avec un schéma standard appliqué sur un maillage 2D nonadmissible. Pour citer cet article: A. Name1, A. Name2, C. R. Acad. Sci. Paris, Ser. I 340 (2005).

\section{Version française abrégée}

On s'intéresse dans [6] à la résolution de l'équation de Poisson dans un domaine mince bi-dimensionnel, caractérisé par un petit paramètre $\varepsilon$. On applique la méthode (MAPDD) de décomposition asymptotique partielle de domaine, introduite dans [5], pour obtenir un problème 1D-2D simplifié. Un schéma hybride 1D-2D de type volumes finis a été proposé et une estimation d'erreur a été obtenue en traitant

Email address: canon@univ-st-etienne.fr (Marie-Claude Viallon). 
séparément les parties uni-dimensionnelle et bi-dimensionnelle du domaine réduit par la MAPDD. L'estimation d'erreur dépend du pas d'espace et de $\varepsilon$, elle n'est pas optimale par rapport au pas d'espace $[7]$.

On considère ici la résolution de l'équation de Poisson $(\mathrm{P})$, définie section 3 , sur le domaine $\Omega_{\varepsilon}$ représenté Figure 1a. On rappelle [6] le problème (PPD), obtenu par la MAPDD, qui est posé sur un domaine tronqué en $x=\delta$ (Figure 1b). On rappelle [5] que l'écart entre les solutions de ces deux problèmes peut être rendu aussi petit que nécessaire en choisissant une valeur de $\delta$ adaptée (Théorème 3.1). Le schéma numérique pour la résolution de (PPD) est rappelé en (1). On note $h$ le pas d'espace.

Dans cet article, on définit (Définition 5.1) une norme $H^{1}$ discrète sur l'ensemble $X(\mathcal{T})$ des fonctions $W$ constantes par mailles sur le domaine 1D-2D. L'originalité de cette norme est le rôle que joue $W_{0}$, combinaison convexe des valeurs de $W$ de part et d'autres de l'interface entre la partie 1D et la partie $2 \mathrm{D}$ du domaine. Le résultat principal obtenu est une majoration, en cette norme $H^{1}$ discrète, de l'erreur commise en approchant la solution du problème (PPD) par le schéma hybride. On obtient (Théorème $5.2)$ une estimation d'erreur en $O(h)$.

On s'intéresse ensuite au schéma TPFA [3] pour résoudre le problème $(\mathrm{P})$ sur un maillage 2D nonadmissible de $\Omega_{\varepsilon}$. Ce maillage, représenté Figure 2a, est construit en conservant sur $\Omega_{\varepsilon}^{\prime}$ le même type de maillage admissible que précédemment, et en choisissant de grosses mailles rectangulaires dans la partie restante (qui était réduite à un axe 1D précédemment). L'estimation d'erreur est en $O(\sqrt{\varepsilon})$. On constate que les performances de ce schéma sont très similaires avec celles du schéma hybride. On observe numériquement un ordre 2 de convergence en $h$, et un ordre $1 / 2$ de convergence en $\varepsilon$ dans les deux cas.

\section{Introduction}

We consider in [6] the Poisson equation in a two-dimensional thin domain (its thickness is a small parameter). Then we use the method of asymptotic partial domain decomposition (MAPDD), introduced in [5], to obtain a simplified 1D-2D problem. The MAPDD reduces the dimension of the domain in its main part, keeping the initial formulation in the remaining part and prescribing asymptotically precise conditions on the interface. In section 3, we remind the 2D Poisson equation, the associated 1D-2D problem, and the error estimate between the solutions of these two problems. An hybrid 1D-2D finite volume scheme is recalled in section 4 . We derive an error estimate. The global error estimate is splited into three parts : the error related to the $1 \mathrm{D}$ part, the one related to the $2 \mathrm{D}$ part and the interface error. We use classical norms to estimate each of these terms. The global error estimate, in $L^{2}$ norm, is reminded in section 3, it is not optimal in terms of the step of the mesh [7].

As distinct from [6,7], we propose in section 5, a global approach to manage the theoretical study. We define a $H^{1}$ discrete norm for functions defined in a structure that consists of a two-dimensional part and a one-dimensional part. This allows to improve significantly the error estimate. The main result is given in Theorem 5.2 : we state a first order error estimate, in this specific $H^{1}$ norm, in terms of the step of the mesh.

In section 6, we compare our original hybrid scheme with the TPFA scheme [3] set in a 2D domain that we mesh by choosing large 2D cells in the part of the domain which is $1 \mathrm{D}$ with the application of the MAPDD. We obtain atypical cells along the interface that give a non-admissible mesh. We prove that the hybrid scheme converges with an order $\frac{1}{2}$ in terms of the small parameter, as it is the case for the TPFA scheme on this particular non-admissible mesh [1]. 


\section{The model problem and dimension reduction with the MAPPD.}

Let us consider the Poisson equation (P) set on $\Omega_{\varepsilon}$, where $\Omega_{\varepsilon}$ is the open bounded subset of $\mathbb{R}^{2}$ described in Figure 1a. We denote $\gamma_{1}=\left\{(1, y) \in \mathbb{R}^{2} \mid y \in\left(-\frac{\varepsilon}{2}, \frac{\varepsilon}{2}\right)\right\}, \gamma_{2}=\left\{(0, y) \in \mathbb{R}^{2} \mid y \in(-\varepsilon, \varepsilon)\right\}$, and $\gamma_{3}=\partial \Omega_{\varepsilon} \backslash\left(\gamma_{1} \cup \gamma_{2}\right)$.

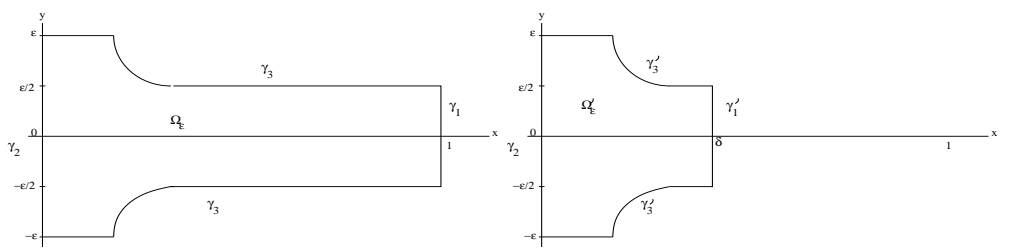

Figure 1. The initial domain (a) and the decomposed domain (b).

Figure 1. Le domaine initial (a) et le domaine partiellement décomposé (b).

$(P)\left\{\begin{array}{l}\Delta u_{\varepsilon}(x, y)=f(x), \quad(x, y) \in \Omega_{\varepsilon} \\ u_{\varepsilon}=0, \text { on } \gamma_{1} \cup \gamma_{2} \\ \frac{\partial u_{\varepsilon}}{\partial n}=0, \text { on } \gamma_{3}\end{array} \quad(P P D)\left\{\begin{array}{l}v^{\prime \prime}(x)=f(x), x \in(\delta, 1), v(1)=0 \\ \triangle u(x, y)=f(x),(x, y) \in \Omega_{\varepsilon}^{\prime} \\ \frac{\partial u}{\partial n}=0, \text { on } \gamma_{3} \cap\{x \leq \delta\}=\gamma_{3}^{\prime}, \quad u=0, \text { on } \gamma_{2} \\ u(\delta, y)=v(\delta), y \in\left(-\frac{\varepsilon}{2}, \frac{\varepsilon}{2}\right), \text { i.e. }(\delta, y) \in \gamma_{1}^{\prime} \\ v^{\prime}(\delta)=\frac{1}{\varepsilon} \int_{-\frac{\varepsilon}{2}}^{\frac{\varepsilon}{2}} \frac{\partial u}{\partial x}(\delta, y) d y\end{array}\right.\right.$

We assume that $f$ is a regular function that does not depend on $y$. The MAPDD reduces the dimension in space on the right of the domain $\Omega_{\varepsilon}$. The $2 \mathrm{D}$ part of the new domain Figure $1 \mathrm{~b}$ is called $\Omega_{\varepsilon}^{\prime}$. Let us denote $D_{\varepsilon}=\Omega_{\varepsilon}^{\prime} \cup\{(x, 0), x \in(\delta, 1)\}$. The so-called partially decomposed problem is the hybrid 1D-2D problem (PPD) set on $D_{\varepsilon}$. We denote $u^{d}(x, y)=u(x, y), x<\delta$, and $v(x), x \geq \delta$, for $(x, y) \in \Omega_{\varepsilon}$, the solution of (PPD). We assume $u^{d}$ is regular. We remind [5]:

Theorem 3.1 For any $J$, there exist $M$, independent of $\varepsilon$, such that if $\delta=M \varepsilon|\ln (\varepsilon)|$, then $\left\|u_{\varepsilon}-u^{d}\right\|_{H^{1}\left(\Omega_{\varepsilon}\right)}=O\left(\varepsilon^{J}\right)$.

The hybrid finite volume scheme that is used to solve (PPD) is recalled in section 4 . Let denote by $h$ the step of the mesh. We prove [6]:

Theorem 3.2 Let $u_{\mathcal{T}}^{d}$ the approximate finite volume solution of (PPD). Then, if $\delta=M \varepsilon|\ln (\varepsilon)|$ and $\frac{h|\ln (\varepsilon)|}{\varepsilon}$ tends to zero when $\varepsilon, h$ tend to zero, we have $\left\|u_{\varepsilon}-u_{\mathcal{T}}^{d}\right\|_{L^{2}\left(\Omega_{\varepsilon}\right)}=O\left(\sqrt{\frac{h}{\varepsilon}} \delta\right)+O\left(\varepsilon^{J}\right)$.

\section{The numerical scheme}

Let us remind the hybrid numerical scheme. In order to define a numerical approximation $u_{\mathcal{T}}^{d}$ of $u^{d}$, let us define a 1D mesh of the interval $(\delta, 1-\delta)$. We choose $N_{1} \in \mathbb{N}^{*}$, and $N_{1}+1$ distinct and increasing values $x_{i+1 / 2}, i=0, \ldots, N_{1}$, such that $x_{1 / 2}=\delta, x_{N_{1}+1 / 2}=1-\delta$. We let $I_{i}=\left(x_{i-1 / 2}, x_{i+1 / 2}\right)$, and $h_{i}=x_{i+1 / 2}-x_{i-1 / 2}, i=1, \ldots, N_{1}$. Then we choose $N_{1}$ points $x_{i}$ in $I_{i}$. Given $x_{0}=\delta, x_{N_{1}+1}=1-\delta$, $h_{i+1 / 2}=x_{i+1}-x_{i}, i=0, \ldots, N_{1}$.

We assume $\Omega_{\varepsilon}^{\prime}$ is polygonal. Let $\mathcal{T}$ be a 2 D mesh of $\Omega_{\varepsilon}^{\prime}$ such that $\overline{\Omega_{\varepsilon}^{\prime}}=\cup_{K \in \mathcal{T}} \bar{K}, K$ being open polygonal convex subsets of $\Omega_{\varepsilon}^{\prime}$. We denote by $\mathcal{P}$ a family of points of $\Omega_{\varepsilon}^{\prime}, \mathcal{P}=\left(x_{K}\right)_{K \in \mathcal{T}}$. We will refer to $x_{K}$ as the center of $K$. 
Let $\mathcal{E}$ be the family of edges $\sigma$ of the control volumes. Let $\mathcal{E}_{K}$ be the family of edges of K. Let $\mathcal{E}_{\text {int }}=\left\{\sigma \in \mathcal{E}, \sigma \not \subset \partial \Omega_{\varepsilon}^{\prime}\right\}$. Let $d_{\sigma}$ be the distance between $x_{K}$ and $x_{L}$ if $\sigma \in \mathcal{E}_{\text {int }}, \sigma=\sigma_{K / L},(K \neq L)$, and $d_{\sigma}$ be the distance between $x_{K}$ and $\sigma$, if $\sigma \in \mathcal{E}_{K}$ and if $\sigma \subset \partial \Omega_{\varepsilon}^{\prime}$.

Let $m(K)$ be the area of $K$, for any $K \in \mathcal{T}$, and $m(\sigma)$ be the length of $\sigma$, for any $\sigma \in \mathcal{E}$.

We assume the 2D mesh of $\Omega_{\varepsilon}^{\prime}$ is admissible, that is $x_{K} \neq x_{L}$ and the straight line through $x_{K}$ and $x_{L}$ is orthogonal to $\sigma_{K / L}$ (see [3]). So the two point approximation $F_{K, \sigma}(1)$ of the normal flux through $\sigma_{K / L}$ is consistent. Let $h$ be the size of the global 1D-2D mesh, $h<\varepsilon$.

The approximation $u_{\mathcal{T}}^{d}$ of $u^{d}$ is defined by $u_{\mathcal{T}}^{d}(x, y)=u_{\mathcal{T}}(x, y)=u_{K},(x, y) \in K, K \in \mathcal{T}$, and $v_{\mathcal{T}}(x)=$ $v_{i}, x \in\left(x_{i-1 / 2}, x_{i+1 / 2}\right), i=1, \ldots, N_{1}$. The scheme is given by :

$$
\left\{\begin{array}{l}
F_{i+1 / 2}-F_{i-1 / 2}=h f_{i}, f_{i}=\frac{1}{h} \int_{x_{i-1 / 2}}^{x_{i+1 / 2}} f(x) d x, i=1, \ldots, N_{1}, \\
F_{i+1 / 2}=\frac{v_{i+1}-v_{i}}{h_{i+1 / 2}}, i=0, \ldots, N_{1}, v_{N_{1}+1}=v(1)=0, \\
\sum_{\sigma \in \mathcal{E}_{K}} F_{K, \sigma}=m(K) f_{K}, f_{K}=\frac{1}{m(K)} \int_{K} f, \forall K \in \mathcal{T} \\
F_{K, \sigma}= \begin{cases}\frac{m(\sigma)}{d_{\sigma}}\left(u_{L}-u_{K}\right), & \forall \sigma \in \mathcal{E}_{i n t}, \text { if } \sigma=\sigma_{K / L} \\
\frac{m(\sigma)}{d_{\sigma}}\left(-u_{K}\right) & , \forall \sigma \subset \gamma_{2}, \sigma \in \mathcal{E}_{K} \\
\frac{m(\sigma)}{d_{\sigma}}\left(v_{0}-u_{K}\right) & , \forall \sigma \subset \gamma_{1}^{\prime}, \sigma \in \mathcal{E}_{K} \\
0 \quad, \forall \sigma \subset \gamma_{3}^{\prime}, \sigma \in \mathcal{E}_{K} & (c)\end{cases}
\end{array}\right.
$$

We notice that $v_{0}$ is an auxiliary unknown.

\section{Error estimate solving directly the partially decomposed problem}

Here, a direct study, entirely different from [6] allows to derive a more accurate error estimate.

Definition 5.1 We define $X(\mathcal{T})$ the set of the functions from $D_{\varepsilon}$ to $\mathbb{R}$ which are constant over each control volume of the mesh. Let $W \in X(\mathcal{T}), W(x, y)=W_{K},(x, y) \in K, K \in \mathcal{T}$, and $W_{i}, x \in\left(x_{i-1 / 2}, x_{i+1 / 2}\right), i=$ $1, \ldots, N_{1}$. We introduce the norm

$\|W\|_{1, \mathcal{T}}=\left(\sum_{\sigma \in \mathcal{E}_{i n t}, \sigma \subset \gamma_{2} \cup \gamma_{1}^{\prime}} m(\sigma) d_{\sigma}\left(\frac{D_{\sigma} W}{d_{\sigma}}\right)^{2}+\varepsilon \sum_{i=0}^{N_{1}} \frac{\left(W_{i+1}-W_{i}\right)^{2}}{h_{i+1 / 2}}\right)^{1 / 2}$

where $D_{\sigma} W=\left\{\begin{array}{l}\left|W_{K}-W_{L}\right|, \text { if } \sigma \in \mathcal{E}_{\text {int }}, \sigma=\sigma_{K \mid L} \\ \left|W_{K}\right|, \text { if } \sigma \subset \gamma_{2}, \sigma \in \mathcal{E}_{K}, \\ \left|W_{K}-W_{0}\right|, \text { if } \sigma \subset \gamma_{1}^{\prime}, \sigma \in \mathcal{E}_{K} .\end{array} \quad\right.$ and where $W_{N_{1}+1}=0$

with $W_{0}=\left(\frac{W_{1}}{h_{1 / 2}}+\frac{1}{\varepsilon} \sum_{\sigma \subset \gamma_{1}^{\prime}, \sigma \in \mathcal{E}_{K}} \frac{m(\sigma)}{d_{\sigma}} W_{K}\right)\left(\frac{1}{h_{1 / 2}}+\frac{1}{\varepsilon} \sum_{\sigma \subset \gamma_{1}^{\prime}} \frac{m(\sigma)}{d_{\sigma}}\right)^{-1}$.

The $\|\cdot\|_{1, \mathcal{T}}$ norm is a discrete $H^{1}$ norm on the domain $D_{\varepsilon}$.

Let $e_{\mathcal{T}} \in X(\mathcal{T}), e_{\mathcal{T}}(x, y)=e_{K}=u\left(x_{K}\right)-u_{K},(x, y) \in K, K \in \mathcal{T}$, and $e_{i}=v\left(x_{i}\right)-v_{i}, x \in\left(x_{i-1 / 2}, x_{i+1 / 2}\right), i=$ 
$1, \ldots, N_{1}$. We define $e_{0}$ as $W_{0}$ above, and we prove the key result of this paper :

Theorem $5.2\left\|e_{\mathcal{T}}\right\|_{1, \mathcal{T}}=O\left(\frac{h}{\sqrt{\varepsilon}}\right)$.

Sketch of the proof. Let consider the equations (1a). We substract the equations obtained by integrating $v^{\prime \prime}=f$ on each $1 \mathrm{D}$ cell and by integrating $\Delta u=f$ over each control volume $K \in \mathcal{T}$. We take into account the consistency errors on the diffusion flux to introduce $e_{\mathcal{T}}$. We multiply each expression by the value of $e_{\mathcal{T}}$ on the suitable cell, and we sum on the cells. In the resulting sum, we get $\left\|e_{\mathcal{T}}\right\|_{1, \mathcal{T}}$, some small terms and the terms coming from the consistency error on the diffusion flux on the interface. The latter do not tend to zero when $h$ tends to zero. If we denote the consistency errors on the diffusion flux by $R_{K, \sigma}$ when $\sigma \subset \gamma_{1}^{\prime}, \sigma \in \mathcal{E}_{K}$, and by $R_{1 / 2}$ on the right side of the interface, we prove that $\sum_{\sigma \subset \gamma_{1}^{\prime}, \sigma \in \mathcal{E}_{K}} m(\sigma) R_{K, \sigma}-\varepsilon R_{1 / 2}=0$. This allows simplifications in the final sum and leads to the result.

\section{The comparison with non matching grids}

In this section $\mathcal{T}$ denote the non-admissible mesh of $\Omega_{\varepsilon}$ described in Figure 2a. To constitute this mesh, we keep an admissible mesh of $\Omega_{\varepsilon}^{\prime}$, and we choose an admissible regular rectangular mesh in the part of $\Omega_{\varepsilon}$ which was previously reduced in one dimension. So the edges on the interface do not satisfy the orthogonality condition. Let $u_{\mathcal{T}}^{n a}$ denote the approximate solution of $(\mathrm{P})$ obtained using the
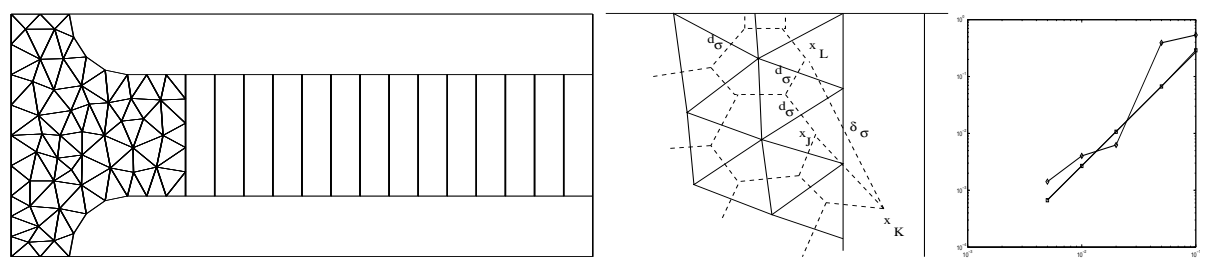

Figure 2. The non-admissible mesh (a). Atypical edge along the interface (b). Convergence orders (c).

Figure 2. Le maillage non-admissible (a). Une arête atypique le long de l'interface (b). Ordres de convergence (c).

TPFA scheme on this non admissible grid, $u_{\mathcal{T}}^{n a}(x, y)=u_{K}^{n a},(x, y) \in K, K \in \mathcal{T}$. The numerical flux [1] is $F_{K, \sigma}=\frac{m(\sigma)}{\delta_{\sigma}}\left(u_{L}^{n a}-u_{K}^{n a}\right), \sigma \in \mathcal{E}_{i n t}, \sigma=\sigma_{K / L}$, instead of (1b).

If $\sigma \not \subset \gamma_{1}^{\prime}$, then $d_{\sigma}=\delta_{\sigma}$. If $\sigma \subset \gamma_{1}^{\prime}$, the edge is atypical (see Figure 2b) since the straight line through $x_{K}$ and $x_{L}$ is not orthogonal to $\sigma_{K / L}$, so the flux is not consistent [4].

According to [1] we deduce that $\left\|e_{\mathcal{T}}^{n a}\right\|_{L^{2}\left(\Omega_{\varepsilon}\right)}=O\left(\frac{h}{\sqrt{\varepsilon}}\right)+O(\varepsilon)=O(\sqrt{\varepsilon})$, since $h<\varepsilon$, where $e_{\mathcal{T}}^{n a}(x, y)=$ $u_{\varepsilon}\left(x_{K}\right)-u_{\mathcal{T}}^{n a}\left(x_{K}\right), \forall(x, y) \in K, K \in \mathcal{T}$.

This yields $\left\|u_{\varepsilon}-u_{\mathcal{T}}^{n a}\right\|_{L^{2}\left(\Omega_{\varepsilon}\right)}=O(\sqrt{\varepsilon})$. We obtain the same result for the hybrid scheme by using a discrete Poincaré inequality and the Theorem 5.2. This is confirmed by the numerical tests (see the second line of the table 1).

In the numerical experiments, $f$ is chosen so that the solution of $(\mathrm{P})$ is known [7]. On Figure 2c we give the error curves function of $h$ of three schemes : the hybrid scheme ( $\square)$, the TPFA scheme on a 2D admissible grid of $\Omega_{\varepsilon}(\diamond)$, and the TPFA scheme on the non-admissible grid (Figure 2a) (-). We see that the straight lines obtained with the hybrid scheme and the TPFA scheme on the non-admissible grid are almost superimposed. We notice some instabilities with the standard TPFA scheme. The slope of the lines gives the order of convergence (logarithmic scale). The numerical convergence orders in $L^{2}$ norm are given in the first line of the table 1 . This superconvergence is classically observed for the TPFA scheme in 2D for the Poisson equation [2], whereas the theoretical convergence order is lower [3]. We conclude 


\begin{tabular}{|c|c|c|c|}
\hline \multicolumn{4}{|c|}{ scheme $1 \mathrm{D} / 2 \mathrm{D}$ hybrid TPFA non-admissible TPFA standard } \\
\hline$h$ & 2.0209 & 1.9964 & 2.1975 \\
\hline$\varepsilon$ & 0.4996 & 0.50723 & - \\
\hline
\end{tabular}

Table 1

Error in $L^{2}$ norm. Erreur en norme $L^{2}$.

the TPFA scheme on the 2D non-admissible grid and the 1D-2D hybrid scheme give similar results.

\section{Conclusion}

Thanks to Theorem 5.2, we obtain a first order error estimate, in terms of the space step, for the hybrid 1D-2D finite volume scheme. This proves that the scheme is as accurate as the standard TPFA scheme on an admissible 2D mesh [3], even if the dimension of the problem has been reduced on a part of the domain. We use a specific $H^{1}$ norm to establish the theoretical estimate in a set of functions defined on a 1D-2D domain. As distinct from [6,7] in which we just use classical 1D norms and 2D norms.

\section{References}

[1] R. Cautrés, R. Herbin and F. Hubert, The Lions domain decomposition algorithm on non matching cell-centered finite volume meshes, IMA Journal of Numerical Analysis Vol. 24, pp. 465-490, (2004).

[2] K. Domelevo, and P. Omnes. A finite volume method for the Laplace equation on almost arbitrary two-dimensional grids. M2AN 39 (6) : 1203-1249, 2005

[3] R. Eymard, T. Gallouët, R. Herbin. Finite Volume Methods. Handbook of Numerical Analysis, P.G. Ciarlet and J.L. Lions eds, (2000).

[4] I. FAILlE. A control volume method to solve an elliptic equation on a 2D irregular meshing. Comp. Meth. Appl. Mech. Engrg., 100,pp. 275-290, (1992).

[5] G.P. PAnasenko. Method of asymptotic partial decomposition of domain. Mathematical Models and Methods in Applied Sciences. 8(1):139-156,1998.

[6] G.P. PAnasenko And M.-C. Viallon. The finite volume implementation of the partial asymptotic domain decomposition. Applicable Analysis an International Journal. 87(12) : 1397-1424, 2008.

[7] G.P. PAnasenko and M.-C. Viallon. Error estimate in a finite volume approximation of the partial asymptotic domain decomposition. to appear in MMAS. 